\title{
FUNCTION THEORY FOR A BELTRAMI ALGEBRA
}

\author{
B. A. CASE \\ Department of Mathematics \\ The Florida State University \\ Tallahassee, Florida 32306 \\ (Received July 16, 1984)
}

ABSTRACT. Complex functions are investigated which are solutions of an elliptic system of partial differential equations associated with a real parameter function. The functions $f$ associated with a particualr parameter function $g$ on a domain $D$ form a Beltrami algebra denoted by the pair $(D, g)$ and a function theory is developed in this algebra. A strong conformality property holds for all functions in a $(D, g)$ algebra. For $g \equiv|z|=r$ the algebra $(D, r)$ is that of the analytic functions.

KEY WORDS and PHRASES: Elliptic system of partial differential equations, Beltrami algebra, dilation. 1980 AMS SUBJECT CLASSIFICATION CODES: 30G30; 30G20; 30 C60.

\section{INTRODUCTION.}

The idea of defining classes of functions which are generalizations of the analytic functions by means of systems of first order partial differential equations goes back at least to a paper of Picard in 1891 [1]. The solutions of that system were of the type later called "pseudo-analytic functions of the second kind" by Bers [2] where for $f=u+i v$

$$
\begin{aligned}
& u_{x}=a v_{x}+b v_{y} \\
& -u_{y}=b v_{x}-a v_{y}
\end{aligned}
$$

where $a, b, c$ and $d$ are appropriate real-valued functions. The relationship of solutions of the elliptic system

$$
\begin{aligned}
& u_{x}=a v_{x}+b v_{y} \\
& -u_{y}=c v_{x}+a v_{y}
\end{aligned}
$$

where $a, b, c$ and $d$ are real valued and $b c-a^{2}=1$, to those of system (1.1) is well developed in papers by Caldwell, [3, 4]

Two current research areas related to the early investigations of elliptic systems are the theory of ouasiconformal mappings and function theoretic methods in partial differential equations. Works from the latter point of view which have 
extensive references are Vekua, [5] and Gilbert and Buchanan, [6]. Ahlfurs discusses, in his introduction to [7], analytic theory generalizations and rationale concerning quasiconformal mappings in particular. Examples from the algebras of functions which we consider are found in very different areas of investigation, indicating the possibility of an interesting and useful function theory. (e.g., Concerning quasiconformal mappings see [8], p. 71; topological analysis, [9] and [10], p. 198; .l1iptic systems of partial differential equations, 151, p. 131.)

The functions considered are solutions of a Beltrami differential eouation with complex dilatation at each point $z$ dependent on a real parameter function $g$. A generalized derivative $f_{g}^{\prime}$ is defined. Necessary and sufficient conditions for existence of $f_{g}^{\prime}$ include the Beltrami equation which reduces to the Cauchy-Riemann equations for analvtic $f$. Both re $f$ and $i m f$ satisfy the same second order partial differential equation with analytic antecedent the Laplace equation.

Although the available generalization of the Laplace eouation and its applications are of interest in anvelliptic system, the parallel is not always so direct. (e.g., In the system of 1111 a different generalized system holds for each of re $f$, im $f$.)

For functions in algehra $(n, g)$, a generalized conformality holds which is invariant in the algebra, that is, for given $z$ and two fixed arcs the angle between the image arcs is the same for all functions in the algebra. The generalized line integral $g \int f$ shares in modified form much of the theory of the ordinary complex line integral. Among some applications in the last section is a function continuous on the finite complex plane, in a $(D, g)$ algebra on $\phi-\{(0,0)\}$, $K$-quasiconformal on bounded sets, and which maps $\phi$ onto a fixed open disk.

2. NOTATION AND NFFINITIONS.

Complex valued functions of one complex variable $z=r e^{i \theta}=x+i y$ are denoted $\mathrm{f}$ and $\mathrm{ref}=\mathrm{u}, \mathrm{im} \mathrm{f}=\mathrm{v},|\mathrm{f}|=\rho$ and arg $\mathrm{f}=\phi$. Partial derivatives are denoted by subscriots. Standard definitions used are:

$$
\begin{aligned}
& f_{z}=e^{-i \theta}\left(f_{r}-(i / r) f_{\theta}\right), \\
& f_{z}=e^{i \theta}\left(f_{r}+(i / r) f_{\theta}\right), \\
& J=\left|f_{z}\right|^{2}-\left|f_{z}\right|^{2} .
\end{aligned}
$$

The complex plane is denoted $\phi$ and a domain $D \subset \phi$ is an open connected set (and, later in the paper, is required to be simply connected). The classes $c^{n}\left|C^{\infty}\right|$ comprise the functions with continuous partial derivatives of $n$ lalll orders. Usage is generally consistent with $\{121$ and $\{131$ as concerns classical analysis; $[8], 17]$ and $[14]$ as concerns auasiconformal functions; and 115$]$ as concerns functional analysis.

The derivative $f_{g}^{\prime}$ defined here is the limit of a generalized difference quotient involving the parameter function $g$ which reduces to the difference quotient for analytic functions. For $f \in C^{\prime}$ in a domain, existence of the limit of this generalized difference ouotient is shown in $[16]$ to be necessary and sufficient for our defining condition ( 2.2 below).

DEFINITION 1. A parameter function $g$ in the following discussion is a real valued positive function of $|z|=r$ for $z \in D$, such that $g^{\prime}=d g / d r$ exists and is continuous on $D$. 
DEFINITION ?. The ordered pair $(r, q)$ represents the set of complex valued humeomorphisms $f \in C^{2}(D)$ satisfying

$$
\mathrm{f}_{\bar{z}}=\frac{(1-(\mathrm{g} / \mathrm{r}))}{(1+(\mathrm{g} / \mathrm{r}))} e^{\mathrm{i} 2 \theta_{\mathrm{f}_{z}}}
$$

For such functions the generalized derivative is defined

$$
f_{g}^{\prime}=e^{-i \theta_{f}} \text {. }
$$

A function is called g-analytic at a point $z$ when there is a parameter function $g$ so (2.2) holds on some domain containing $z ; f_{g}^{\prime}$ is called the g-derivative.

The Reltrami equation (2.2) is equivalent to a generalized Cauchy-Riemann condition. When stated in rectangular coordinates in the form (1.2) the functions $a, b, c$ are: $a=x y\left(g^{2}-r^{2}\right) / g r^{3} ; b=\left(x^{2} r^{2}+y^{2} g^{2}\right) / g r^{3}$;

$c=\left(y^{2} r^{2}+x^{2} g^{2}\right) / g r^{3}$. For computational convenience we state that condition as a system in polar form

$$
\begin{aligned}
& (i m f)_{\theta}=g(\text { ref })_{r} \\
& (\text { re } f)_{\theta}=-g(i m f)_{r}
\end{aligned}
$$

or,

$$
\mathrm{f}_{\theta}=\mathrm{ig} \mathrm{f}_{\mathrm{r}} \text {. }
$$

nEFINITION ?. Let $f$ be complex valued and continuous on (piecewise) differentiable arc $\gamma$ on which $r \neq 0$. A generalized line integral dependent on parameter function $g$ is defined by:

where $\omega=\exp \int_{1}^{r} d r / g$.

$$
\begin{aligned}
& \int_{\gamma} f=\int_{\gamma}(\omega / g)(u \cos \theta-v \sin \theta) d r-(v \cos \theta+u \sin \theta) d \theta \\
&+i \int_{\gamma}(\omega / g)(v \cos \theta+u \sin \theta) d r+(u \cos \theta-v \sin \theta) d \theta
\end{aligned}
$$

The properties of the lemma follow frum the definitions in a straightforward manner.

LEMMA 1 .

a) When $f \in(n, g), J=(g / r)\left|f_{r}\right|^{2}$.

h) The constant functions are in each set $(D, Q)$ and are the only functions in $\left(n, g_{1}\right) \cap\left(D, g_{2}\right), g_{1} \neq g_{2} ; f_{g}^{\prime} \equiv 0$ for a constant function.

c) If $f \in(n, g)$ and $f_{g}^{\prime} \equiv n$, then $f$ is constant. Also, $f$ is constant if either re $f$, im $f,|f|$ or arg $f$ is identicallv constant.

d) If $f \in(n, g)$ and $c$ is a complex number, then $c f \in(n, g)$.

e) If $f, h \in(D, g)$ then the sum, product and reciprocals are in $(D, g)(1 / f$ for $f \neq n)$ and the generalized derivatives are:

$$
\begin{aligned}
& (f+h)_{g}^{\prime}=f_{g}^{\prime}+h_{g}^{\prime}, \\
& (f h)_{g}^{\prime}=f_{g}^{\prime} h+h_{g}^{\prime} f, \\
& (1 / f)_{g}^{\prime}=-f_{g}^{\prime} /(f)^{\prime} .
\end{aligned}
$$

f) If $f, h \in(D, g)$ such that $f_{g}^{\prime} \equiv h_{g}^{\prime}$ on $D$, then $f-h$ is a constant function on $D$.

g) Tf nonconstant $f \in(D, g)$ and $h \in(D, r)$ then $h \circ f \in(D, g)$. 
h) $(D, g)$ is an algebra over $\phi$. (The multiplicative unit is $f \equiv 1$.)

For $g \equiv r,(D, g)$ is the set of functions analytic on $D$, and (2.3) and (2.5) are, respectively, the usual derivative and 1 ine integral. The reouirement in Definition 1 that the function $g$ be positive is equivalent to reauiring that the Jacobians for functions $f \in(n, g)$ be non-negative and hence that the functions be sense preserving. This is cunsistent with practice in the larger auasiconformal class llthough, is rhire, miny of these resulta hold for sense reversing functions. nthar conditions on $f$ or $g$ in the definitions mav he weakened in some annlicationc. Sae 1171 , 141 fur careful discussion of topological and anasiconfurmal prupurties of Reltrani sustems.

\section{MAPPINS PROPE,RTIFC}

Functions which are solutions of the Reltrami equation (2.) are locallv cuasicunformal. In darticular, at $z \in \Pi$ the dilatation auotient is given

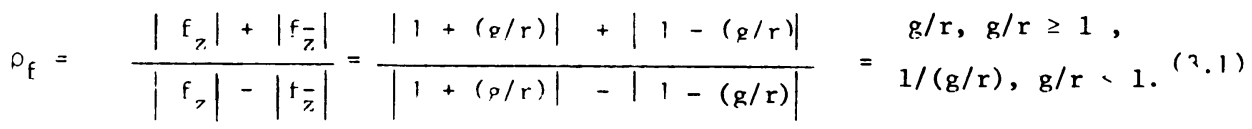

The dilatatwo guotient is bounded on every compact subset of $r$ and, if it is boundet on $n$ then $f$ is a regular k-nuasiconformal manping for

$$
\mathrm{K} \geq \operatorname{sun}_{\mathrm{z} \in \mathrm{D}} \max \{\rho / \mathrm{r}, \mathrm{r} / \mathrm{g}\}
$$

A strong generalioation if conformality holds for g-analvtic functions. Given a point $z$ and the parameter function $g$, the angle between the images of two fixed arcs is the same whichever $f \in(n, g)$ maps the ares. This reduces to angle preservation in the confurmal case. It is more specific than the inequality availahle for the larger class of arasiconformal mappings in general. (see ligl, Theorem 4, which is an eauivalence to auasi-conformalitv.)

THFORFM 1. Arcs $\gamma_{1}$ and $\gamma_{2}$ intersect at $z$ where they have tangents which make angles respectively of $\alpha$ and $\beta$ with the real axis and of $\sigma$ and $\tau$ with the radial direction through $z$. Tf $f \in r^{l}$ is g-analytic in a domain cuntaining $z$ then the angle $\psi^{\prime}$ between $f\left(\gamma_{1}\right)$ and $f\left(\gamma_{2}\right)$ is determined by:

$\sin \psi^{\prime} / \cos \psi=((g / r) \sin (\beta-\alpha))\left(\left(\cos ^{2} \theta+(g / r)^{2} \sin ^{2} \theta\right) \cos \alpha \cos \beta\right.$

$+\left(\sin ^{2} \theta+(g / r) \cos ^{2} \theta\right) \sin \alpha \cos \beta+\cos \theta \sin \theta\left(1-(g / r)^{2}\right)\left(\sin (\alpha+\beta) 1^{-1}\right.$

$=\left((g / r)(\sin (\tau-0)) /\left(\cos \left(\cos \tau+(g / r)^{2} \sin \sigma \sin \tau\right)\right.\right.$.

Converselv, if $f \in c^{l}, T>n$, and (3.2) holds on some domain containing $z$ where $\&$ satisfies nefinition 1 , then $f$ is g-analytic at $z$.

PROAF. The swcond of the forms of (?.)) is most convenient to derive. The first then follows, since $\sigma=\alpha+\Pi-\theta, \tau=\beta+\Pi-\theta$. Let $\sigma^{\prime}$ be the angle made by $f\left(\gamma_{1}\right)$ at the point $f(z)$ with the ratial firection through $f(z)$. Manipulating algebraicallv with allowable angles, we have

$$
\begin{aligned}
\frac{\sin \sigma^{\prime}}{\cos \sigma^{\prime}}= & \rho(\mathrm{d} \phi / \mathrm{d} \rho)=\frac{r \cos \sigma \phi_{r}+\sin \sigma \phi_{\theta}}{r \cos \sigma \rho_{r}+\sin \sigma \rho_{\theta}} \\
= & \frac{\left(r \cos \sigma v_{r}+\sin \sigma v_{\theta}\right) u-\left(r \cos \sigma u_{r}+\sin \sigma u_{\theta}\right) v}{\left(r \cos \sigma v_{r}+\sin \sigma v_{\theta}\right) v-\left(r \cos \sigma u_{r}+\sin \sigma u_{\theta}\right) u}
\end{aligned}
$$


and, using similar expressions for $\tau$, $\tau^{\prime}$ write

$\frac{\sin \psi^{\prime}}{\cos \psi^{\prime}}=\frac{\sin \left(\tau^{\prime}-\sigma^{\prime}\right)}{\cos \left(\tau^{\prime}-\sigma^{\prime}\right)}$

$$
\begin{aligned}
& =\frac{\left(r \cos \sigma u_{r}+\sin \sigma u_{\theta}\right)\left(r \cos \tau v_{r}+\sin \tau v_{\theta}\right)-\left(r \cos \sigma v_{r}+\sin \sigma v_{\theta}\right)\left(r \cos \tau u_{r}+\sin \tau u_{\theta}\right)}{\left(r \cos \sigma v_{r}+\sin \sigma v_{\theta}\right)\left(r \cos \tau v_{r}+\sin \tau v_{\theta}\right)+\left(r \cos \sigma u_{r}+\sin \sigma u_{\theta}\right)\left(r \cos \tau u_{r}+\sin \tau u_{\theta}\right)} \\
& =\frac{r \operatorname{im}\left(\bar{f}_{r} f_{\theta}\right)(\sin (\tau-\sigma)}{\left(r^{2}\left|f_{r}\right|^{2} \cos \sigma \cos \tau+\sin \sigma \sin \tau\left|f_{\theta}\right|^{2}+r(\sin (\sigma+\tau)) r e\left(\bar{f}_{r} f_{\theta}\right)\right.} .
\end{aligned}
$$

Since $f \in(D, g)$ by hypothesis, substitution using (2.4) gives (?.2).

Conversely, if $f$ is a function for which the expressions (3.2), (3.3) are equivalent for all arcs, then

$r^{2}\left(g\left|f_{r}\right|^{2}-i m\left(\bar{f}_{r} f_{\theta}\right)\right)+r\left(g\left(r e\left(\bar{f}_{r} f_{\theta}\right)\right)(\tan \sigma+\tan \tau)\right)+\left(\left(g\left|f_{\theta}\right|^{2}-g{ }^{2} i m\left(\bar{f}_{r} f_{\theta}\right)(\tan \sigma \tan \tau)\right)=0\right.$

and hence we have the system

$$
\begin{aligned}
g\left|f_{r}\right|^{2}- & i m\left(\bar{f}_{r} f_{\theta}\right)=0 \\
\operatorname{re}\left(\bar{f}_{r} f_{\theta}\right) & =0
\end{aligned}
$$

which is equivalent to the complex form of $(2.4):$ since $J>0$, $f$ is sense: preserving, hence $f$ is g-analytic.

DFFINITION 4. If $\mathrm{f} \in(D, g)$ and $\mathrm{f}$ satisfies (3.2) locally for all $z \in D$, then $f$ is g-conformal on $D$.

For all functions in a given $(n, g)$ algebra, the g-conformality gives the angle hetween the images of intersecting arcs depending on the tangents to the arcs themselves and the point at which they intersect. In the algebra of analytic functions $(D, r)$ the relation is not only also independent of the intersection point but, reducing to angle preservation, is the same for all pairs of arcs $\left(\gamma_{1}, \gamma_{2}\right)$ with the same angle between them. The similarity to the analytic case is less obvious in the case of the effect of the g-analytic function on arc length. Where ds and dS are elements of arc in the $z$ and $f(z)$ olanes, respectively,

$$
d S^{2}=\left|f_{g}^{\prime}\right|^{2}\left(d r^{2}+g^{2} d \theta^{2}\right) \text {. }
$$

The modulus of the generalized derivative retains position as a magnification factor, although not in the Fuclidean metric.

4. PROPERTIES OF THE FUNCTION THEORY

In addition to some theory of the generalized derivative (2.3) and integral (2.5), we give a generalized harmonicity relation which is satisfied bv both re $f$ and $i m f$ for $f$ in a $(n, g)$ algebra. It is helpful to first construct two examples which are the most general functions in a $(D, g)$ algebra under certain conditions.

From now on, we assume a domain $D$ is a simply connected.

EXAMPLE 1. If at $z \in \mathrm{D}$

$$
f(z)=\lambda(r) \exp \left(i\left(c_{l} \theta+c_{2}\right)\right)
$$

where $c_{1}, c_{2}$ are real constants, $c_{1} \neq 0$, and nonconstant real valued $\lambda(r) \epsilon$ $C^{2}(D)$, then $f$ is $g$-analytic at $z$ and $g=c_{1} \lambda / \lambda^{\prime}$. On the other hand, when $f_{1}$ 
is a g-analytic function of form (4.1) then

$$
f_{1}(r)=\operatorname{exn}\left(c_{1} \int d r / g+i\left(c_{1} \theta+c_{2}\right)\right) \text {. }
$$

EXAMPLE 2. If $\mathrm{f}_{2} \in(\mathrm{D}, \mathrm{g})$ and $\left(\mathrm{re} \mathrm{f}_{2}\right)_{\theta} \equiv 0$, then there exist real constants $c_{1}, c_{2}$ and a branch of arg $z=\theta$ such that

$$
f_{2}(z)=c_{1} \int d r / g+i\left(c_{1} \theta+c_{2}\right) ;
$$

when $h \in(n, g)$ and $(r e h)_{r} \equiv 0$ then $h \equiv i f_{2}$, where $f_{2}$ is as (4.3). The statement folluws since (im $\left.f_{?}\right)_{\theta}$ is independent of $r$ and (re $\left.f_{?}\right)_{r}$ is indenendent of $\theta$, so that there exists real $c_{1}$ such that $\left(i m f_{?}\right)_{\theta}=$ $g\left(r e f_{2}\right)^{r}=c_{1}$.

We consider two relationships hetween the example functions of (4.2) and (4.3). Clearly $f_{1}=h \circ f_{2}$ where $h$ is the exponential function. Ry lemma 19 on composition of functions $f_{1}$ is g-analvtic if and only if $f_{2}$ is g-analvtic. Also, tho g-derivative of $f_{2}$ is given by $\left(f_{2}\right)_{g}^{\prime}=\left(c_{1} / g\right) e^{-i \theta}$ which is $\rightarrow$ function of the form of $(1.1)$ and hence is $g_{1}$-analvtic for $g_{1}=g / g^{\prime}$. Now, in general, if we require the function of form (4.1) to be in $\left(n, g_{1}\right)$ we note that $f_{g}^{\prime}=$ $\lambda^{\prime} \exp \left(i\left(\left(c_{1}-1\right) \theta+c_{2}\right)\right)$ and $g_{1}=c_{1} \lambda / \lambda^{\prime}$. It follows by mathematical induction that the bth derivative exists under appropriate conditions. (See Theorem 3h.)

Theorems 2 and 3 discuss the existence of higher order derivatives.

THEORFM 2. Let $f \in(D, \lambda)$ and $z \in D$, then $f^{\prime}$ is g-analvtic at $z$ for real positive function $g=\delta$ only if at least one of the following conditions hulds:

a) There is a real number $k$ such that $\lambda(r)=r+k$; for this case $\delta=r+k$.

h) There exists real valued differentiable $h(\theta)$ such that $(i m f)_{r}=$ $h(\theta)(r, f)_{r}$.

E) Either $(r e f)_{\theta}$ or $(r e f)_{r}$ is zero.

PROnF. Let $f=u+i v$; then $r e f_{\lambda}^{\prime}=\cos \theta u_{r}+\sin \theta v_{r} ; i m f_{\lambda}^{\prime}=\cos \theta v_{r}-\sin \theta u_{r}$. Requiring that (2.4) hold for some real $\lambda(r)$ gives the matrix eouation

$$
\left[\begin{array}{rr}
\cos \theta & \sin \theta \\
\sin \theta & -\cos \theta
\end{array}\right]\left[\begin{array}{ll}
{ }^{{ }_{r}} & { }^{u_{r r}} \\
{ }^{r}{ }_{r} & v_{r r}
\end{array}\right]\left[\begin{array}{l}
\lambda^{\prime}-1 \\
\lambda-\delta
\end{array}\right]=\left[\begin{array}{l}
0 \\
0
\end{array}\right] \text {. }
$$

If the third matrix is the zero matrix then we have a). When $u_{r}$ or ${ }_{r}$ is zero, we have c). (See Fxample 2, (4.?).) otherwise, the requirement that $u_{r} v_{r r}{ }^{-v_{r}} u_{r r} \equiv$ 0 implies the relation of b).

THEORFM 3.

a) If $f \in(D, g)$ for $g(r)=r+k$, then $f_{r+k} \in(D, g)$. If $f \in c^{\infty}(D)$, all orders of higher derivatives exist in $(n, r+k)$ and are defined by

$$
f_{r+k}=e^{-i n \theta} \frac{\partial^{n} f}{\partial r^{n}} .
$$

b) If $f \in\left(n, g_{1}\right)$ is of the form $f=\lambda \exp \left(i\left(c_{1} \theta+c_{2}\right)\right.$ where $g_{1}=$ $c_{1} \lambda / \lambda^{\prime}$, then the nth derivative exists for

$$
\begin{aligned}
& n \leq \min \left\{c_{1}, \max \left\{d \in \text { integers } \mid \lambda^{(d)} \text { exists }\right\}, c_{1}>n,\right. \\
& \max \left\{d \in \text { integers } \mid \lambda^{(d)} \text { exists }\right\}, c_{1}<n .
\end{aligned}
$$


In particular, where $g_{n}=\left(c_{1}-n+1\right) \lambda^{(n-1)} / \lambda^{(n)}$, then

$$
\left(\left(f_{g_{1}}^{\prime}\right) g_{2}^{\prime} \ldots\right)_{g_{n}}^{\prime}=\lambda^{(n)} \exp \left(i\left(\left(c_{1}-n\right) \theta+c_{2}\right)\right) \text {. }
$$

(When $c_{1}<n$ and $\lambda \in C^{\infty}$ all orders of higher derivatives exist.)

c) If nonconstant $f \in(D, \lambda)$ with $(r e f)_{\theta}=0$ or $(r e f)_{r}=0$ then the first derivative exists and reduces to case $b$ ). (See the discussion following Fxample 2.)

PRחnF. a) and b) can be proved using mathematical induction.

If $f$ is in an algebra $(D, g)$, then both re $f$ and im $f$ satisfy the sam.. second order partial differential equation which is the Laplace ecuation for analvtic $f$. In a simply connected domain construction of coniugate g-analytic functions f 1 ! lows.

THFORFM 4. If $f \in(n, g)$, re $f$ and im $f$ rach satisfy

$$
2 \phi_{r r}+g^{\prime} \phi_{r}+(1 / g) \phi_{\theta \theta}=n \text {. }
$$

DEFTNITION 5. Real valued $\phi(r, \theta) \in c^{2}$ satisfuing (4.5) is caller g-harmonic. Real functions $u(r, \theta)$ and $v(r, \theta)$ which satisfy (2.4) art colled g-coniugates.

THFORFM 5. Tf $\phi$ is g-harmonic in $n$, then $\phi$ has at least one $q-$ cun iugate.

PROMF. Without loss of generality, let 11 satisfv (4.5) and consider

$$
v(r, \theta)=\int_{0}^{r}-u_{\theta}\left(r, \theta_{0}\right) d r / g+\int_{0}^{\theta} g u_{r}(r, \theta) d \theta
$$

where $z_{0}$ is a fixed point $z_{0}=r_{0} \exp \left(i \theta_{0}\right)$. It is immediate that $v_{\theta}(r, \theta)=$ g' $r(r, \theta)$ and we also compute

$$
v_{r}(r, \theta)=-u_{\theta}\left(r, \theta_{0}\right) / g+\int_{\theta}^{\theta}\left(\lambda u_{r r}(r, \theta)+\lambda u_{r}(r, \theta)\right) d \theta
$$

which after substitution of (4.5) reduces to $v_{r}=-u_{\theta} / g$, satisfying (2.4) for $\mathrm{f}=\mathrm{u}+\mathrm{iv}$.

Examination of Definition 3 of the g-integral as an Riemann path integral involving the function $g$ shows that the usual operational properties regarding multiples and path components continue to hold. We derive a few useful properties of the generalized line integral; in particular these are analogues of Cauchy's Theorem and path independence, Morera's Theorem, and some convergence theorems of the Weierstrass type. Domains are simply connected.

THEOREM 6. If $f \in(D, g)$ and $\gamma$ is a simple closed curve homologous to 0 in D then $\mathrm{g} / \mathrm{f}=0$.

PROOF. Using the polar form of Green's Theorem, re ${ }^{g} f f$ and $i m{ }^{g} / f$ each reduce to zero after substitution of the generalized Cauchy Riemann conditions (2.4). The stronger form of Cauchy's Theorem, allowing exceptional points in the domain, is developed in $[16], \mathrm{P} .62-66$.

COROLLARY 1. When $f \in(D, g)$, then $\int_{a}^{g} f$ is independent of the path joining $a$ and $b$ in $D$.

PROOF. If two paths form $a$ to $b$ in $D$ do not cross or cross a finite number of times, the result follows by Theorem 5 and the definitions and operational properties of the path integrals. If the paths cross infinitely often a limiting process is available. (See [13], p. 315). 
THEOREM 7. Let $f \in(D, g)$ and $z_{0}$ be fixed in $D$, and define $F=\int_{z_{0}}^{z} f$; then $F \in(D, g)$ and further
$F_{g}^{\prime}=\left(\left(\exp \int_{1}^{r} \mathrm{dr} / g\right) / g\right) f$.

PROOF. Since the generalized integral is path independent we apply properties of line integrals in the plane to compute the partial derivatives of the real and the imaginary parts of the integral function F. System (2.4) is satisfied, so the derivative function is given by $(2.3)$ as $F_{g}^{\prime}=e^{-i \theta}\left({ }_{z}^{g} \int_{0}^{z} f\right)_{r}$ which is equivalent to $(4.6)$.

We thus demonstrate for the generalized case the differentiability of the integral. The analytic case $g(r) \equiv r$ is seen by (4.6) as necessary (as well as sufficient) that $F_{g}^{\prime} \equiv f$.

THFOREM 8. Let $\mathrm{f} \in \mathrm{C}^{\mathrm{l}}$ in $\mathrm{D}$ and $\mathrm{g}$ satisfy Definition 1 . If $\int_{\gamma} f=0$ for every simple closed curve $\gamma$ in $D$, then $f$ is g-analytic in $D$.

PROOF. In (2.5) both the real and imaginary parts of the generalized integral are represented by real line integrals $\int_{Y}(P d r+Q d \theta)$ where $P$ and $Q$ are both in $\mathrm{C}^{\mathrm{l}}$. Since by the hypothesis we have independence of path for these integrals, $P_{\theta}=Q_{r}$, or:

$$
\begin{aligned}
& (1 / g)\left(u_{\theta} \cos \theta-v_{\theta} \sin \theta\right)=-v_{r} \cos \theta-u_{r} \sin \theta, \\
& (1 / g)\left(v_{\theta} \cos \theta+u_{\theta} \sin \theta\right)=u_{r} \cos \theta-v_{r} \sin \theta .
\end{aligned}
$$

Algebraic manipulations of this system show (2.4) for $f=u+i v$.

Before showing, some Weirstrass-type convergence results, we need a bounding relation on the generalize integral which depends on inequalities for real line integrals.

LEMMA 2. When $\gamma$ is rectifiable

where $K_{g}=4 \max _{z \in \gamma}\left(\left(\exp _{1} \int_{\gamma}^{r} \mathrm{dr} / g\right)\left(1+\max _{z \in \gamma}|1 / g|\right)\right), M=\max _{z \in \gamma}|f|, L=$ length of $\gamma$.

THEOREM 9. If $f_{n}$ is continuous on regular arc $\gamma$ for each $n$, and the sequence $\left\{\mathrm{f}_{n}\right\}$ converges uniformly to $f$ on $\gamma$ then

$$
\lim _{n \rightarrow \infty} \int_{\gamma}^{g} f_{n}={ }_{\gamma}^{g} f
$$

PROOF. Since $f$ is continuous there is an $N$ such that for $n>N$

by Lemma 2 .

$$
\left|\int_{\gamma}^{g} f_{n}-\int_{\gamma}^{g} f\right|=\left|\int_{\gamma}^{g}\left(f_{n}-f\right)\right| \leq \varepsilon^{\prime} K_{g} L<\varepsilon
$$

THEOREM 10. If for all $n, f_{n} \in C^{2}$ is g-analytic on open set $A$ and $\left\{f_{n}\right\}$ converges uniformly to $f$ on compact subsets of $A$, then $f$ is g-analytic on $A$. PROOF. For $z \in A$ there is a disk containing $z$ with its closure in $A$. He 
show $f \in(D, g)$ on such disks, hence $f$ is g-analytic for $z \in A$. Let $Y$ be any simple closed curve in a disk contained in $A$. Then $g / f_{n}=0$ for each $n$ by Theorem 6. By Theorem 9, $g / f=0$ hence Theorem 8 gives g-analyticity in the disk. COROLLARY 2. If to the hypothesis of Theorem 10 is added, for $f_{n}=u_{n}+i v_{n}$ that $\left\{u_{n}\right\}$ converges uniformly to $u$ and $\left\{v_{n}\right\}$ converges uniformly to $v$ where $f=u+i v$, then $f_{g}^{\prime}=\lim _{n \rightarrow \infty}\left(f_{n}\right){ }_{g}^{\prime}$.

PRONF. We now have $\lim _{n \rightarrow \infty}\left(u_{n}\right)_{r}$ and $\lim _{n \rightarrow \infty}\left(v_{n}\right)_{r}=v_{r}$ hence there is an $N$ such that for $n>N$

$$
\left.\left.\left|\left(f_{n}\right)_{g}^{\prime}-f_{g}^{\prime}\right|=\mid e^{-i \theta}\left(\left(\left(u_{n}\right)_{r}+i\right) v_{n}\right)_{r}\right)-\left(u_{r}+i v_{r}\right)\right) \mid<\varepsilon .
$$

A version of the argument principle and of Cauchy's Integral Formula are available from the theory of Beltrami's equation. For completeness we show the latter for g-analytic functions.

THEOREM 11. If $f \in(D, g)$ and $\gamma$ is a simple closed curve homologous to 0 in $D$, and $z_{0}$ is in the interior of $\gamma$, then

$$
f\left(z_{o}\right)=\frac{1}{2 \pi i} \int_{\gamma} \frac{f(z) d z}{z-z_{o}}+\frac{1}{2 \pi} \iint_{\text {Int } \gamma} \frac{(g-r) e^{i 2 \theta} f_{g}^{\prime}(z) d r d \theta}{z-z_{o}} .
$$

see [5], p. 24-25 and p. 91 for discussion of more general cases.

\section{APPLICATIONS}

One interesting example of a g-analytic function with domain $\mathbf{p}-\{(0,0)\}$ and locally quasiconformal with continuous extension at the isolated boundary point $(0,0)$, and $K$-quasiconformal on bounded sets, maps the finite plane onto a fixed open disc.

EXAMPLE 3. For fixed $\rho$, real and such that $0<\rho<\infty$, define

$$
f(z)=\left\{\begin{array}{cl}
\left(2 \rho e^{i \theta} \tan ^{-1} r\right) / \pi, & z \neq 0, \\
0 & , z=0 .
\end{array}\right.
$$

The function satisfies (2.2) for $g=\left(1+r^{2}\right) t^{-1} r$ and maps the finite plane onto the open disc $|f(z)|<\rho$.

Concentric annuli may be g-conformally mapped whatever the proportions of their radii.

EXAMPLE 4. For annuli: $A_{1}=0<r_{1}<|z|<r_{2}<\infty$,

$$
A_{2}=0<R_{1}<|z|<R_{2}<\infty \text {, }
$$

let

$$
f(z)=e^{i \theta}\left(R_{2}\left(r-r_{1}\right)-R_{1}\left(r-r_{2}\right)\right) /\left(r_{2}-r_{1}\right) \text {. }
$$

Then $f: A_{1} \rightarrow A_{2}$ homeomorphically and $f$ is g-analytic for $g=r+\left(\left(R_{1} r_{2}-R_{2} r_{1}\right) /\left(R_{2}-R_{1}\right)\right)$.

The convergence properties of the g-integral allow a comparison to the general quasiconformal situation. When the generalized functions satisfying the Beltrami equation (2.2) are quasiconformal in a domain (see Section 3), they are of the type called regular quasiconformal. Although the uniform convergence of a sequence of $\mathrm{K}-$ 
quasiconformal mappings to a homeomorphism implies that the $1 \mathrm{imit}$ mapping is also $\mathrm{K}-$ quasiconformal, in the case of a sequence of regular $\mathrm{K}$-quasiconformal mappings the limit mapping need not be regular. (See [8], p. 17-19.) For g-analytic regular Kquasiconformal functions, the $1 \mathrm{imit}$ mapping is $\mathrm{g}$-analytic.

COROLlaRY 3. A Beltrami algebra $(D, g)$ is uniformly closed.

\section{REFERENCES}

1. PICARD, E. Sur un système d'équations aux dérivés partielles, C.R. Acad. Sci. Paris 112 (1891) 685-689.

2. BERS, L. Theory of Pseudo-analytic Functions, New York University Lecture Notes, 1963.

3. CALDWELL, W.v. Some relationships between Bers and Beltrami systems and 1 inear elliptic systems of partial differential equations, Can. J. Math. 17 (1965) 627-642.

4. CALDWELL, W.V. The dilatation of harmonic quasi-conformal functions, Proc. Amer. Math. Soc. 40 (1973) 205-208.

5. VEKUA, I.N. Generalized Analytic Functions, Addison Wesley, Reading, 1962.

6. GILBERT, R.P., and BUCHANAN, J.L. First Order Elliptic systems, Academic Press, New York, 1983.

7. AHLFORS, L.V. Lectures on Quasiconformal Mappings, D. Van Nostrand, Princeton, 1966.

8. LEHTO, O. and VIRTANEN, K.I. Quasiconformal Mappings in the Plane, Second Edition, Springer-Verlag, New York, 1973.

9. EGGLESTON, H.G. and URSELL, H.D. On the lightness and strong interiority of analytic functions, J. London Math Soc. 27 (1952) 260271 .

10. WhYBURN, G. T. Analytic Topology, Amer. Math. Soc. Colloquium Publ. Vol. 28, Providence, 1942.

11. BERS, L. and GELBART, A. On generalized Laplace transformations, Annals of Math. 48 (1947) 342-357.

12. AHLFORS, L.V. Complex Analysis, Second Edition, McGraw-Hill Book Company, New York, 1966 .

13. KAPLAN, W. Advanced Calculus, Second Edition, Addison-Wesley Press, New York, Reading, 1977.

14. LAWRYNOWICZ J. and KRZYZ, J. Quasiconformal Mappings in the Plane: Parametric Methods, Springer-Verlag, New York, 1983.

15. RUDIN, W. Functional Analysis, McGraw-Hill Book Company, New York, 1973.

16. CASE, B.A. On non-analytic functions related to a system of partial differential equations, University Microfilms \# 70-9333 (Ph.D. thesis).

17. CALDWELL, W.V. An algebra of 1 ight interior functions, Duke Math J. 34 (1967) 225-230.

18. AGARD, S.B. and GEHRING, F.W. Angles and quasiconformal mappings, Proc. London Math. Soc. 3 14A (1965) 1-21. 


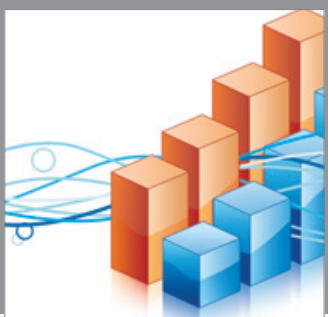

Advances in

Operations Research

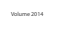

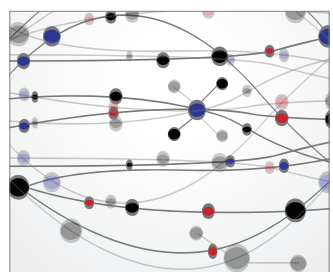

\section{The Scientific} World Journal
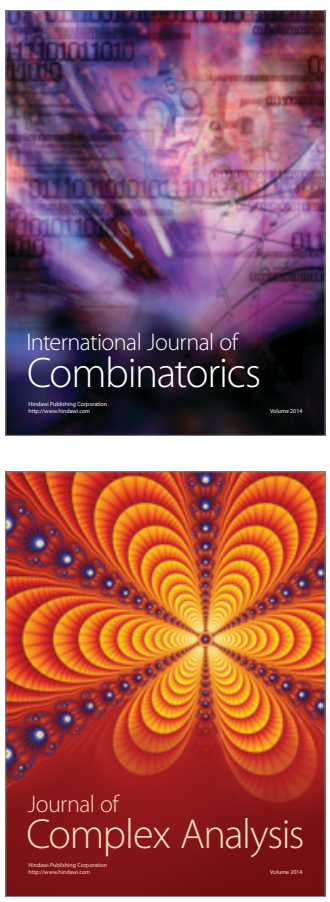

International Journal of

Mathematics and

Mathematical

Sciences
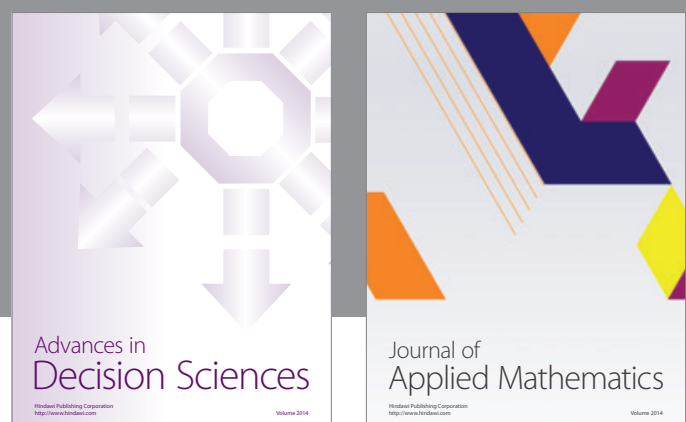

Journal of

Applied Mathematics
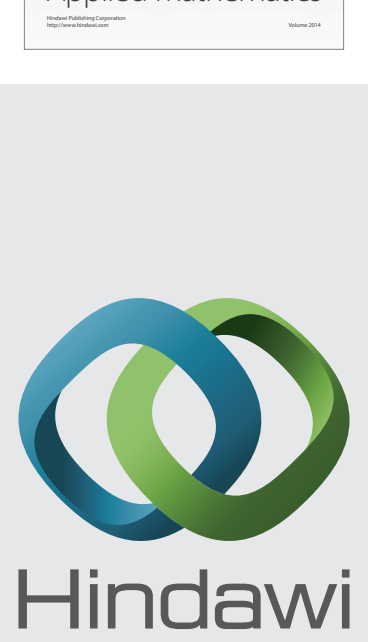

Submit your manuscripts at http://www.hindawi.com
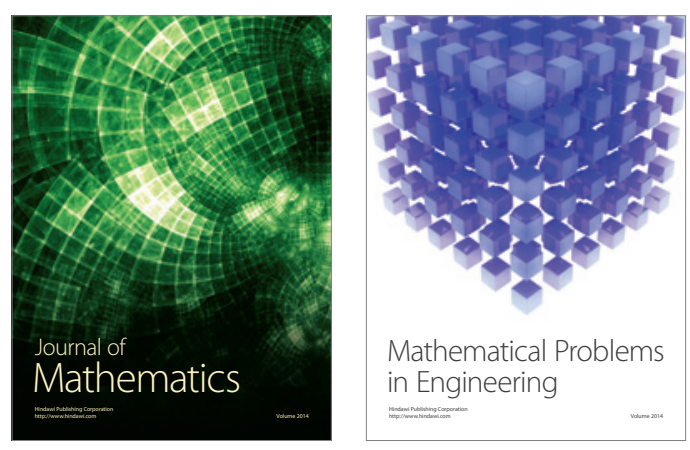

Mathematical Problems in Engineering
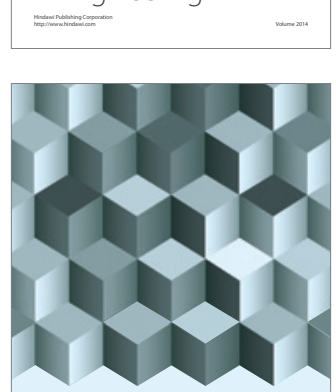

Journal of

Function Spaces
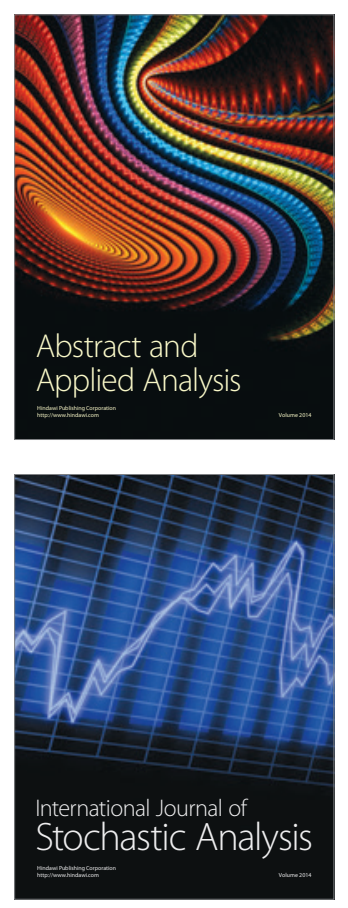

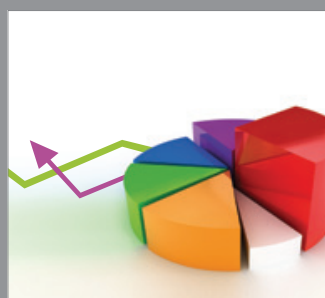

ournal of

Probability and Statistics

Promensencen
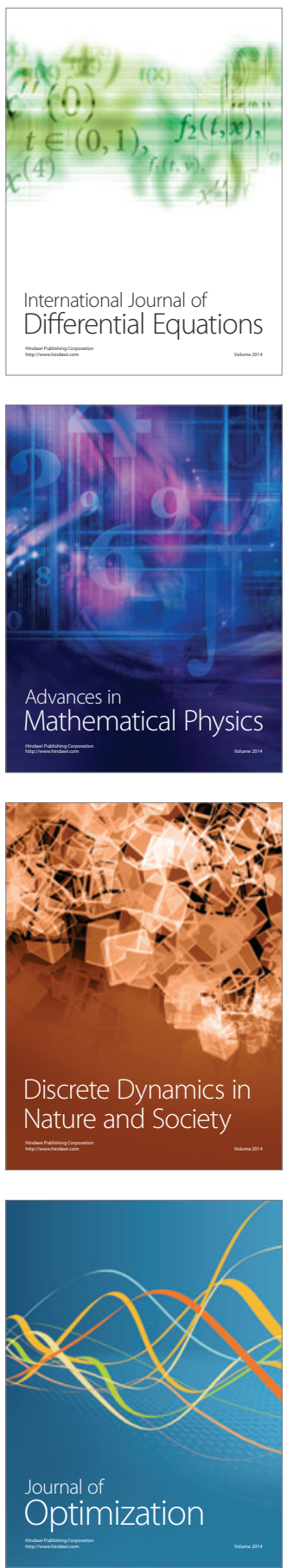